\title{
Developmental delay-facial dysmorphism syndrome due to MED13L deficiency
}

INSERM

\section{Source}

INSERM. (1999). Orphanet: an online rare disease and orphan drug data base. Developmental delay-facial dysmorphism syndrome due to MED13L deficiency. ORPHA:369891

A rare, genetic, multiple congenital anomalies/dysmorphic syndrome characterized by varying degrees of intellectual disability, global developmental delay (notably with severe speech and language impairment), muscular hypotonia, and facial dysmorphism (i.e. broad forehead, bitemporal narrowing, upslanting palpebral fissures, low-set ears, flat nasal bridge, bulbous nose and, variably, macrog lossia). Highly variable additional features include cardiac defects (including persistent foramen ovale, ventricular septal defects, tetralogy of Fallot), coordination problems, seizures, abnormal growth parameters (including microcephaly, low birth and postnatal weight), and brain morphology anomalies (such as ventriculomegaly and myelination defects). 